\title{
Investigating the Relationship Between Neighborhood Experiences and Psychiatric Distress for Individuals with Serious Mental Illness
}

\author{
Bret Kloos • Greg Townley
}

Published online: 31 July 2010

(C) Springer Science+Business Media, LLC 2010

\begin{abstract}
The present study examined the relationships between how research participants experienced their neighborhood, their neighborhood social climate, and psychological well-being. Participants $(n=525)$ were residents of supported housing programs who used mental health services at one of 17 community mental health centers in South Carolina. Hierarchical regression and mediation analyses were employed to answer research questions. Results suggest that neighbor relations, perceptions of neighborhood safety, and neighborhood satisfaction were significantly associated with perceptions of neighborhood social climate; and neighborhood social climate accounted for a significant amount of the variance in psychiatric distress. Of particular interest, perceptions of neighborhood social climate fully mediated the relationship between the specific reported neighborhood experiences and psychiatric distress. These findings have implications for interventions and policy aimed at promoting integration of individuals with serious mental illness into community settings.
\end{abstract}

\section{Introduction}

Over the past two decades, housing has increasingly been viewed as a critical factor for mental health treatment and rehabilitation of persons with serious mental illness (e.g., Goldman and Morrisey 1985; Carling 1990; Cohen and Somers 1990; Dixon and Osher 1995; Newman 2001). Access to affordable, quality housing is integral to the

B. Kloos $(\bowtie) \cdot$ G. Townley

Department of Psychology, University of South Carolina,

1512 Pendleton Street, Columbia, SC 29208, USA

e-mail: kloos@sc.edu success of persons with serious mental illness living outside of institutional care. These issues have become a concern among mental health professionals because housing instability has been documented to contribute to cycles of increased symptomatology, disability, and exposure to harmful community environments (e.g., Drake et al. 1991; Dickey et al. 1996; Lam and Rosenheck 1999; Rog 2004). Thus, housing has ceased to be solely a social welfare issue, but rather is considered an important component of mental health treatment and policy.

Similarly, in the last 10 years, research on the impact of neighborhood contexts on health and well-being is an increasing area of interest and importance (Kawachi 2002). Numerous studies have assessed the mechanisms through which neighborhood components may influence mental and physical health for children (e.g., Caughy et al. 2003; Evans et al. 2003) adults (e.g., Ross et al. 2000; Silver et al. 2002), the elderly (e.g., Young et al. 2004), and ethnic minorities (e.g., Sampson 2003). However, fewer studies have assessed the impact of neighborhood experiences on the well-being of individuals with serious mental illness (SMI), for whom finding healthy, supportive community environments is a great challenge.

The present study examined perceptions of neighborhood social climate as a potential mediator of the relationship between specific neighborhood experiences and psychiatric distress for people with serious mental illness who live in supported housing (see Fig. 1). In this report, we will (a) discuss neighborhood social climate as a construct that captures the importance of community acceptance and tolerance for individuals with SMI; (b) outline three neighborhood experiences that can be critical to promoting neighborhood social climate and belonging; and (c) examine the potential influence of neighborhood experiences and resultant perceptions of neighborhood 
Fig. 1 Theoretical model of the predicted relationships between neighborhood experiences, neighborhood social climate, and psychological well-being

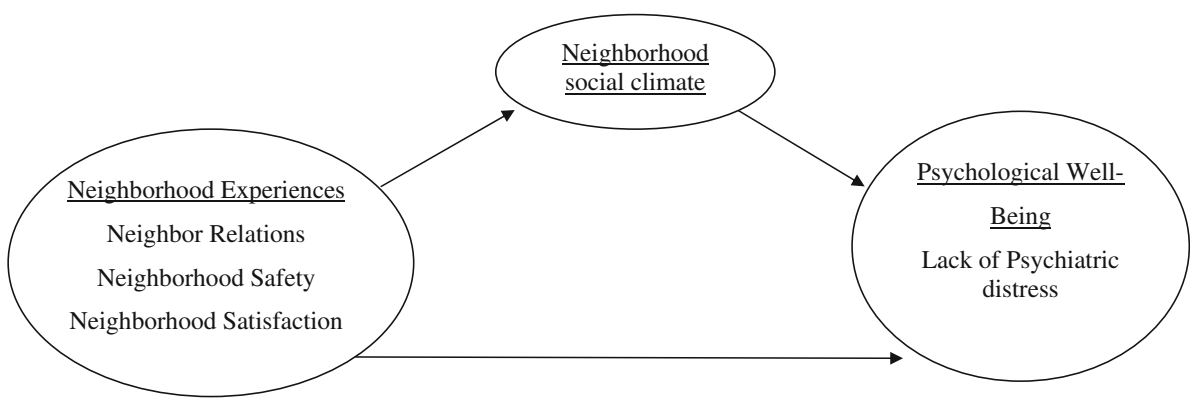

social climate on psychological well-being, as measured by self-reported psychiatric distress.

Developing a Conceptual Framework to Study Neighborhood Experiences for Persons with SMI

The past 25 years have seen profound changes in services for people with SMI. The current trend to supported housing, marked by principles of consumer choice, holding a lease, integration into the community, and flexible mental health services, is replacing long-term institutional treatment and residential treatment facilities (Carling 1993). In the case of supported housing, these apartments are considered "market housing", that is, units that are integrated into community settings and can be rented by a person regardless of disability status ( $\operatorname{Rog} 2004)$. The first advocates of supported housing emphasized models where individuals with mental illness live independently in their own apartments and use supportive, community-based mental health services as needed (Carling 1990; Cohen and Somers 1990). With its emphasis on housing being part of community settings and separate from services (Rog 2004), supported housing presents a challenge to research approaches that have focused on physical aspects of housing or programmatic aspects of housing services. Understanding the experience of community-based living in supported housing requires the development of new approaches for conceptualizing and measuring housing experiences embedded in community settings but apart from services (Yanos 2007). This study draws upon social ecology theory to conceptualize housing experiences related to structural, interpersonal, and community levels of analysis that, taken together, form a multi-dimensional construct of housing environment.

Using a social ecology framework, research can focus on potential resources to promote adaptive functioning and address challenges present in a particular environment that may threaten functioning (Moos 1976; Kloos and Shah 2009). Thus, social ecology theory calls for the systematic conceptualization and assessment of potential sources of support or stressors in the environment of interest. Applying this framework to supported housing research, housing environments can be conceptualized as having three broad domains: (a) the physical environment of housing such as apartments and neighborhood buildings; (b) the social environment of housing such as the neighborhood social climate and perceptions of safety; and (c) interpersonal relationships tied to housing such as those with neighbors and landlords (Kloos and Shah 2009; Moos 1974, 1976). Although these relationships have received little attention in mental health research, policy makers and service providers have consistently emphasized the importance of quality housing and good relationships with landlords and neighbors for housing stability (Carling 1995; Wong and Solomon 2002; Yanos 2007).

The Experience of Belonging and Neighborhood Social Climate

Fiske (2004) identified belonging as one of five core social motives that describe fundamental psychological processes that impact people's thoughts, feelings, and behaviors in relation to other individuals. Belonging to groups helps individuals to survive psychologically and physically (Fiske 2004); and it has been found to correlate with subjective well-being (Baumeister 1991). Feeling a sense of belonging has been documented as being important for individuals with SMI because they appeared to function better in communities that were perceived to be tolerant and supportive (Newman et al. 1994; Wong and Solomon 2002).

A review of the neighborhood literature reveals that there are many ways of conceptualizing and conducting research with neighborhoods (Kloos and Shah 2009; Townley et al. 2009). Some of the most commonly measured constructs are social capital, neighborhood cohesion, and sense of community. Neighborhood social climate is closely related to these, as it measures perceptions of belonging, acceptance, and tolerance of the neighborhood in which one lives or would like to live (Pretty et al. 1996). In her discussion of the similarities between social climate and sense of community, Pretty (1990) suggests that social climate taps more into the support characteristics of environments than does other constructs of neighborhood or 
community. The social climate of a neighborhood may encompass various features, including acceptance and tolerance of diversity, quality of social relationships, security, and belonging.

For individuals with serious mental illness, an important component of the neighborhood social climate would be acceptance and tolerance for psychiatric disability (Nelson et al. 2001; Kloos and Shah 2009; Townley and Kloos 2009). The sense of belonging to a neighborhood may buffer both the negative effects of psychiatric symptomatology and the stigma that often accompanies it (Prince and Prince 2002; Corrigan 2004). Neighborhoods that foster a sense of belonging for people with SMI can provide a context in which socially supportive relationships can be established; these connections are beneficial to physical and mental health (Young et al. 2004). These points echo the seminal work of Faris and Dunham (1939). They argued that individuals with serious mental illness are disproportionately clustered in deteriorated, socially disorganized sections of cities. Residents of disorganized communities find it difficult to develop and maintain positive relationships with family members, neighbors, and local institutions, thus increasing their experience of social isolation and increasing the onset and negative course of mental illness. Although findings such as these are well supported, it is not always clear which neighborhood experiences may contribute to perceptions of belonging, acceptance, or isolation.

\section{Predictors of Neighborhood Social Climate}

In the neighborhood literature, the experiences that appear to be most important in predicting the social climate of the neighborhood are neighbor relations, neighborhood safety, and neighborhood satisfaction. According to Unger and Wandersman (1985), neighboring involves social interaction, symbolic interaction, and the attachment of individuals with people living around them. Neighbor social networks provide social support (e.g., asking for help), instrumental aid (e.g., borrowing or lending tools), and chances for emotional release (e.g., discussing personal problems with neighbors) (Farrell et al. 2004; Prezza et al. 2001). The greater number and quality of these neighbor relations is predictive of a stronger bond to the neighborhood (McMillan and Chavis 1986). Neighboring has been found to be predictive of sense of community and "rootedness" in the neighborhood (Farrell et al. 2004; Prezza et al. 2001). When located in a setting perceived as safe and having resources for addressing needs, such neighboring experiences likely contribute to an opportunity structure in which persons can increase their capacities for connectedness (e.g., enjoying reciprocal social relationships) and citizenship (e.g., enjoying the rights and responsibilities of a democratic society) (Ware et al. 2007, 2008).

In order for individuals to experience acceptance and support in their neighborhoods, it is also important that they feel safe. Research findings show that higher perceptions of neighborhood safety are related to perceptions of neighborhood connection and sense of community (Zeldin and Topitzes 2002; Ziersch et al. 2005), whereas perceptions of higher neighborhood crime are negatively related to sense of community (Martinez et al. 2001). When residents perceive their neighborhoods to be unsafe, feelings of danger may overtake their daily lives, causing them to stay isolated in their homes and refuse to reach out to fellow residents (Zeldin and Topitzes 2002). This has deleterious effects on perceptions of supportive neighborhood social climate, and it appears to be especially problematic for individuals with serious mental illness. Newman (1994) found that, among individuals with SMI, concerns about being victimized by neighborhood crime appeared to overwhelm relatively positive reports about other neighborhood characteristics. The same study concluded that reports of neighborhood crime were $55 \%$ higher for Columbus, $\mathrm{OH}$ residents with serious mental illness compared with all other residents in the city (Newman 1994). These perceptions and concerns of neighborhood violence appear to be well founded; actual reports of violence against individuals with mental illness also appear to be higher. For example, Silver (2002) found that individuals with mental illness were more likely than individuals without a mental illness diagnosis to be victims of violent crime even after controlling for individual- and community-level correlates of violent victimization.

Residential satisfaction at the neighborhood level is a final experience that appears to be relevant to perceptions of supportive neighborhood social climate. Chavis and Wandersman (1990) found block satisfaction and sense of community to be positively related; and Martinez et al. (2001) reported a significant relationship between satisfaction with the neighborhood and sense of community. It is likely that people who are more content with their communities are more likely to become involved in their communities (Grillo et al. 2010) and engage in behaviors and activities that encourage acceptance and tolerance from neighbors. Understanding the connection between neighborhood satisfaction and neighborhood social climate for persons with serious mental illness is of great necessity for programs interested in promoting residential stability in community settings. Data has shown that when individuals with SMI were asked to rate their neighborhoods on a scale of $1-10$, their ratings were as much as $20 \%$ lower than those of all other households on the block (Newman 1994). 


\section{Rationale and Research Questions}

An empirical understanding of factors that can buffer psychiatric distress and enhance adaptive functioning for persons with SMI living in community settings is emerging as a top priority of the community mental health research agenda (Yanos 2007; Townley et al. 2009). Research has demonstrated that a supportive social climate is closely related to overall psychological well-being for individuals with SMI (Prince and Gerber 2005), whereas mental health related stigmatization (i.e., isolation, segregation, lack of belonging) increases depressive-anxiety symptoms and negatively influences social outcomes (Segal et al. 1980; Markowitz 1998). Assessing the impact of neighborhood social climate on psychiatric distress is particularly important for individuals with SMI who live in supported housing because they often live alone-in some instances, for the first time in their lives (Newman et al. 1994). A national evaluation of supported housing found isolation to be a substantial problem facing many mental health consumers, especially since mental health agencies were not yet effective in countering the problem of loneliness (Carling 1993; Yanos 2007). Therefore, it would be helpful for program development and policy decisions to understand how the experience of neighborhoods can influence a supportive social climate for people with SMI, as well as their potential effects on psychological well-being.

Based upon this review and theoretical framework, this study examined the relationships between neighborhood experiences and the well-being of persons with SMI living in supported housing. Neighborhood social climate is proposed as a mediator of the relationship between neighborhood experience and psychiatric distress for people with SMI who live in supported housing. To test this question, three hypotheses were proposed to examine the relationships between three key neighborhood experiences (neighbor relations, neighborhood safety, and neighborhood satisfaction), neighborhood social climate, and psychiatric distress.

Hypothesis 1 It was expected that (a) reports of neighbor relations, (b) perceptions of neighborhood safety, and (c) satisfaction with the neighborhood community would account for significant variance in perceptions of supportive neighborhood social climate. Neighbor relations were expected to be significantly associated with social climate, as the more strongly that residents feel they can trust, depend on, and interact with their neighbors should improve their sense of belonging and acceptance. Similarly, feeling that the neighborhood was a safe place to live would encourage individuals to seek out relationships with neighbors and will increase their perceptions of a supportive neighborhood social climate. Finally, the more satisfied the residents were with the neighborhood, the more likely they would be to seek out social relationships that help to achieve a sense of acceptance.

Hypothesis 2 The second aim of this study was to assess the relationship between neighborhood social climate and psychiatric distress. It was hypothesized that participants who reported greater perceptions of supportive neighborhood social climate would report lower levels of distress.

Hypothesis 3 The third aim of this study was to determine whether neighborhood social climate mediated the relationships between the three neighborhood experiences and psychiatric distress. It was hypothesized that neighborhood social climate would be the primary psychological mechanism responsible for the effect on psychiatric distress. The following example serves to illustrate this hypothesis: residents may interact with their neighbors and report favorable attitudes about them, but it would be the sense of support, belonging, and acceptance that they perceive as a result of these relations (as opposed to the actual interactions and resultant attitudes) that would have the strongest relationship with their reported well-being.

\section{Method}

\section{Participants}

The participants in this study were 527 residents of South Carolina who have serious mental illness and live in supported housing associated with the South Carolina Department of Mental Health (SCDMH). They were recruited from each of the 17 mental health centers throughout South Carolina. Inclusion criteria were that the research participant (a) had a housing subsidy, (b) held a lease for the apartment, and (c) utilized services from the SCDMH. Two of the initial 527 participants were missing all items on the scales used in this report, so they were removed from the analysis.

The remaining 525 research participants were nearly evenly divided by sex; $52 \%$ of the sample was female and $48 \%$ was male. Research participants identified their ethnicity as follows: $50 \%$ were African American, $43 \%$ White, and $7 \%$ reported other ethnic groups. The average age of the participants was 46.18 , with $5 \%$ reported being married or in a similar relationship. Nearly one-third of the sample completed high school or obtained their GED (32\%), roughly another third had at least some college (32\%), and the remaining participants had less than a H.S. education $(36 \%)$. As indicated in SCDMH records, a majority of the participants, $63 \%$, had a thought disorder as their primary diagnosis, $23 \%$ had an affective disorder as a primary diagnosis, $4 \%$ had an anxiety or other disorders as 
a primary diagnosis, and diagnostic information was unavailable for $10 \%$ of the sample. A history of homelessness is a common issue for this population, and $42 \%$ of the sample reported having been homeless at some point in their lives. Finally, 94\% of participants received Supplemental Security Income or Social Security Disability Income, and $19 \%$ were employed at the time of the interview.

\section{Measures $^{1}$}

Neighborhood experiences were assessed with four measures from the Housing Environment Survey (HES) which inquire about the social environment (social climate of neighborhood, sense of belonging, and perceptions of safety) and interpersonal relationships tied to housing (e.g., neighbor) (Kloos and Shah 2009; Wright and Kloos 2007). The scales primarily used a 5-point response set; participants rated how much they agreed or disagreed with statements about each aspect of their housing environment (Table 1).

\section{Relationships with Neighbors}

The HES Neighbor Scale (HES-NBR) measured perceptions of their relationships with neighbors. The scale had seven items which ask about the amount of support and interaction that the individual had with their neighbors. Examples of items include "My neighbors keep an eye on my apartment when I am gone" and "If I needed someone to talk to about a problem, I could talk with one of my neighbors." The internal consistency of the scale was 0.77 and 1 week test-retest correlation for the scale was 0.75 . The sum score was normally distributed.

\section{Perceptions of Safety}

The HES Safety Scale (HES-S) had eight items about frequency of property destruction, burglary, and assaults in the neighborhood. Examples of items include, "How often are people robbed around your building?" and "How often are groups of people just hanging out and causing problems?" The scale had an internal consistency of 0.78 , a 1 week testretest reliability of 0.79 , and was normally distributed.

\section{Residential Satisfaction}

The HES Residential Satisfaction (HES-RS) was a fouritem inventory assessing the degree of satisfaction research participants feel towards their housing, surrounding neighborhood, neighbors, and landlord or property

\footnotetext{
1 Refer to Table 1 for a correlation matrix of the measures under review in this study.
}

manager. Examples of items include "How does your current neighborhood compare to your previous neighborhood?" and "How satisfied are you with your housing as a place to live?"

\section{Neighborhood Social Climate}

The HES Neighborhood Social Climate (HES-NSC) scale measured perceptions of belonging, acceptance, and community tolerance. The HES-NSC had ten items and had a normal distribution. Examples of items include "People in my neighborhood are friendly to everybody no matter what the person's skin color or ethnic background" and "Some people in my neighborhood give me a hard time because of my disability." The internal consistency of the scale was 0.82 and the 1 week test-retest correlation for the scale was 0.71 .

\section{Psychiatric Distress}

Psychiatric distress was assessed using the Brief Symptom Inventory (BSI). The 53-item BSI (Derogatis 1993) asked about psychiatric distress associated with a range of mental health symptoms. The Global Severity Index (GSI) scoring was used in the current analyses. The GSI measured participants' global distress level by combining information about the number of symptoms experienced and their intensity (Derogatis 1993). Response choices for the BSI range from 0 (not at all) to 4 (extremely). The internal consistency for the BSI in this dataset was 0.96.

\section{Symptomatology}

In order to control for the possibility that symptoms were influencing perceptions of neighborhood experiences, we controlled for paranoid and psychotic symptoms. This was done using the Paranoid Ideation and Psychoticism subscales of the Brief Symptom Inventory (BSI) (Derogatis 1993). The Paranoid Ideation dimension represents paranoid behavior, including hostility, suspiciousness, and delusions. The subscale has five items and had an internal consistency of 0.78 in this sample. The Psychoticsim dimension includes items indicative of a withdrawn schizoid lifestyle ranging from mild interpersonal alienation to dramatic psychosis. The subscale had a Cronbach alpha of 0.72 in this sample.

\section{Procedures}

Research staff conducted the 1:1 interviews at the participant's residence or a mental health facility when the residence was not preferred by the participant. Research staff obtained informed consent with the assistance of case managers prior to beginning interviews which were 
Table 1 Correlation matrix of primary measures used in the study

\begin{tabular}{|c|c|c|c|c|c|c|}
\hline Measure & 1 & 2 & 3 & 4 & 5 & \\
\hline 1 & $\begin{array}{l}\text { Neighborhood social } \\
\text { climate }\end{array}$ & - & & & & \\
\hline 2 & Neighbor relations & $0.461 * *$ & - & & & \\
\hline 3 & Neighborhood safety & $0.439 * *$ & $0.194 * *$ & - & & \\
\hline 4 & $\begin{array}{l}\text { Neighborhood } \\
\text { satisfaction }\end{array}$ & $0.494 * *$ & $0.354 * *$ & $0.228 * *$ & - & \\
\hline 5. & Psychiatric distress & $-0.367 * *$ & $-0.213^{* *}$ & $-0.243 * *$ & $-0.184 * *$ & - \\
\hline
\end{tabular}

approved by the Institutional Review Boards of the sponsoring University and the SCDMH. Participants received $\$ 20$ for participating for each interview.

\section{Data Preparation}

Data were entered in a computer database at the time of the interview. Of the 525 participants, $69 \%$ were missing no data for the scales under examination. None of the remaining participants was missing more than $25 \%$ of scale items on any scale. To address the missing data, multiple imputation techniques were used to provide unbiased estimates of parameters and standard errors under the assumption that the data are missing at random (Cohen et al., 2003). We first used SAS PROC MI to generate ten imputation datasets. We then used PROC MIANALYZE to obtain parameter estimates and standard errors combined across the ten imputations. With this procedure, the degrees of freedom for individual parameter estimates vary as the standard errors and degrees of freedom are adjusted based on the amount of missing information for each parameter prior to imputation. Because the missing data can impact each parameter differently, degrees of freedom can also differ for each parameter (Barnard and Rubin 1999; Schafer 1999).

\section{Results}

Neighbor Relations, Neighborhood Safety, and Neighborhood Satisfaction as Predictors of Neighborhood Social Climate

The first hypothesis was tested using a multiple regression analysis. Perceptions of neighborhood social climate was regressed on neighbor relations, neighborhood safety, and neighborhood satisfaction. Results of the first hierarchical regression analysis (see Table 2) showed that the model with demographic and symptom covariates ${ }^{2}$ (race, sex, age, psychotic symptoms, paranoid symptoms), neighbor

\footnotetext{
${ }^{2}$ Please note, the 'sex' variable consists of males and females, with females serving as the reference group for analyses. The 'race' variable is dummy-coded to include African Americans (50\% of the sample) and non-African Americans (Whites, $43 \%$ of the sample, and
}

Table 2 Summary of hierarchical regression analysis for neighborhood experience factors predicting neighborhood social climate $(\mathrm{N}=525)$

\begin{tabular}{lrllll}
\hline Variable & $\beta$ & SE & Partial $R^{2}$ & df & $P$ \\
\hline Block 1 & & & & & \\
Race & -0.04 & 0.04 & 0.003 & 514 & 0.33 \\
Sex & -0.02 & 0.09 & 0.00003 & 520 & 0.81 \\
Age & 0.01 & 0.004 & 0.007 & 520 & 0.09 \\
Paranoid symptoms & -0.12 & 0.05 & 0.04 & 518 & 0.00 \\
Psychotic symptoms & -0.01 & 0.05 & 0.004 & 518 & 0.88 \\
Model $R^{2}$ & 0.09 & & & & \\
Block 2 & & & & & \\
$\quad$ Neighbor relations & 0.30 & 0.04 & 0.11 & 508 & 0.00 \\
Neighborhood safety & 0.27 & 0.04 & 0.09 & 488 & 0.00 \\
Neighborhood satisfaction & 0.24 & 0.04 & 0.06 & 521 & 0.00 \\
Model $R^{2}$ & 0.48 & & & & \\
$\Delta R^{2}$ & 0.39 & & & & \\
$F$ for $\Delta R^{2}$ & 124.18 & & & & 0.00
\end{tabular}

Note: DF's differ because multiple imputation was used to address missing data

relations, neighborhood safety, and neighborhood satisfaction predicting neighborhood social climate was significant: $F(8,517)=62.15, P<0.001$. Each of the individual neighborhood experience factors was significant, and their positive $\beta$ weights revealed that they were positively related to the outcome. The only significant covariate was paranoid symptoms, suggesting that individuals who experienced paranoid thoughts perceived a less positive neighborhood social climate.

The model accounted for $48 \%$ of the variance in participants' perceptions of neighborhood social climate. Examination of the change in $\mathrm{R}^{2}$ values from block 1 (including only the covariates) and block 2 (including both covariates and the neighborhood predictor variables) showed that the block of neighborhood experience factors accounted for $39 \%$ of the variance over and above the effect of the covariates. After partialing out the effects of

Footnote 2 continued

Other race, $7 \%$ of the sample). The non-African American group is the reference group in analyses. 
the covariates and the other predictors, neighbor relations accounted for $11 \%$, neighborhood safety accounted for $9 \%$, and neighborhood satisfaction accounted for $6 \%$ of the variance in neighborhood social climate. Thus, neighbor relations emerged as the strongest predictor of neighborhood social climate scores. This analysis supported the hypotheses that reports of better neighbor relations, reports of higher-levels of neighborhood safety, and higher ratings of neighborhood satisfaction would be associated with greater perceptions of neighborhood social climate for individuals with serious mental illness who live in supported housing.

Neighborhood Social Climate as a Predictor of Psychiatric Distress

Results of the second hierarchical regression analysis (see Table 3) showed that the model with demographic covariates and neighborhood social climate predicting psychiatric distress was significant: $F(6,519)=21.75, P<0.01$. The neighborhood social climate variable was significant, and its negative $\beta$ weight revealed that it was negatively related to psychiatric distress. Thus, higher perceptions of neighborhood social climate were associated with less psychiatric distress.

The model accounted for $44 \%$ of the variance in participants' reports of psychiatric distress. Examination of the change in $\mathrm{R}^{2}$ values from block 1 (including only the covariates) and block 2 (including neighborhood social climate) showed that perceptions of neighborhood social climate accounted for $10 \%$ of the variance over and above the effect of the covariates. This analysis supported the

Table 3 Summary of hierarchical regression analysis for neighborhood social climate predicting psychiatric distress $(\mathrm{N}=525)$

\begin{tabular}{lrllll}
\hline Variable & $\beta$ & $\mathrm{SE}$ & Partial $R^{2}$ & $\mathrm{df}$ & $P$ \\
\hline Block 1 & & & & & \\
$\quad$ Race & -0.08 & 0.19 & 0.001 & 380 & 0.24 \\
Sex & -0.29 & 0.09 & 0.02 & 320 & 0.00 \\
Age & -0.01 & 0.004 & 0.006 & 459 & 0.05 \\
Paranoid symptoms & 0.34 & 0.03 & 0.12 & 452 & 0.00 \\
Psychotic symptoms & 0.41 & 0.03 & 0.14 & 452 & 0.00 \\
Model $R^{2}$ & 0.34 & & & & \\
Block 2 & & & & & \\
Neighborhood social climate & -0.28 & 0.04 & 0.08 & 461 & 0.00 \\
Model $R^{2}$ & 0.44 & & & & \\
$\Delta R^{2}$ & 0.10 & & & & \\
$F$ for $\Delta R^{2}$ & 101.32 & & & & 0.00 \\
\hline
\end{tabular}

Note: DF's differ because multiple imputation was used to address missing data hypothesis that perceptions of positive neighborhood social climate was related to better psychological well-being.

Neighborhood Social Climate as a Mediator of the Relationship Between Neighborhood Experiences and Psychiatric Distress

The final research question was assessed using the fourstep Baron and Kenny (1986) approach to mediation analysis (see Table 4 for parameter estimates and $P$-values for each step ${ }^{3}$ ). Step one satisfied the first requirement of the approach, which is that the predictors (neighbor relations, neighborhood safety, and neighborhood satisfaction) must be significantly related to the outcome (participant reports of psychiatric distress). Step two fulfilled the requirement that the predictors must be significantly related to the mediator (neighborhood social climate). Step three met the requirement that the mediator must be significantly related to the outcome. Finally, and of most importance, step four suggested full mediation because the three neighborhood experience predictors dropped from being significantly related to the outcome at $P<0.01$ to being non-significant when the mediator (neighborhood social climate) was included in the model.

As shown in Table 4 , the $\beta$ weights for each of the neighborhood experience factors dropped substantially in value and were no longer different from zero when neighborhood social climate was included as a mediator. Additionally, the partial $\mathrm{R}^{2}$ values for each predictor were greatly reduced from step one to step four. Neighbor relations, neighborhood safety, and neighborhood satisfaction accounted for very little of the variance in participant reports of psychiatric distress when neighborhood social climate was included in the model.

Although full mediation was suggested by the approach outlined above, it was important to determine whether the indirect, or mediated, effect by which neighbor relations, neighborhood safety, and neighborhood satisfaction were related to psychiatric distress through neighborhood social climate. Table 5 includes the indirect effects $(\alpha \beta), \alpha \beta$ standard errors, and t-test statistics from the Sobel test for each neighborhood experience factor. Each indirect effect was significant at $P<0.01$. The joint effect of neighbor relations, neighborhood safety, and neighborhood satisfaction on psychiatric distress via neighborhood social climate was -0.18 . This formal test further supported the hypotheses that neighborhood social climate mediated the relationship between the neighborhood experience variables and psychiatric distress.

\footnotetext{
${ }^{3}$ Please note, although not reported in the table, each of the steps has controlled for the demographic and symptomatic covariates.
} 
Table 4 Summary of mediation analysis $(\mathrm{N}=525)^{4}$

Note: DF's differ because multiple imputation was used to address missing data

\begin{tabular}{lccccc}
\hline Variable & \multicolumn{1}{l}{ P } & SE & Partial $R^{2}$ & df & $P$ \\
\hline Step 1-neighborhood experiences & predicting & distress \\
Neighbor relations & -0.09 & 0.04 & 0.03 & & \\
Neighborhood safety & -0.11 & 0.04 & 0.04 & 459 & 0.01 \\
Neighborhood satisfaction & -0.08 & 0.05 & 0.02 & 469 & 0.00 \\
Step 2-neighborhood experiences & predicting & social climate & & & \\
Neighbor relations & 0.30 & 0.04 & 0.11 & 508 & 0.00 \\
Neighborhood safety & 0.27 & 0.04 & 0.09 & 488 & 0.00 \\
Neighborhood satisfaction & 0.24 & 0.04 & 0.06 & 521 & 0.00 \\
Step 3-climate predicting distress & & & & & \\
Neighborhood social climate & -0.28 & 0.04 & 0.10 & 461 & 0.00 \\
Step 4-social climate and neighborhood experiences predicting & distress & & \\
Neighborhood social climate & -0.23 & 0.05 & 0.07 & 462 & 0.00 \\
Neighbor relations & -0.004 & 0.05 & 0.00007 & 428 & 0.91 \\
Neighborhood safety & -0.01 & 0.05 & 0.0004 & 438 & 0.43 \\
Neighborhood satisfaction & -0.02 & 0.05 & 0.0006 & 476 & 0.58 \\
\hline
\end{tabular}

Table 5 Indirect effects $(\alpha \beta), \alpha \beta$ standard errors, and $t$-test statistics from the Sobel test for each neighborhood experience

\begin{tabular}{lllll}
\hline Variable & $\alpha \beta$ & $\mathrm{SE}_{\alpha \beta}$ & $t$-test stat & $P$ \\
\hline Neighbor relations & -0.07 & 0.02 & -4.54 & 0.00 \\
Neighborhood safety & -0.06 & 0.02 & -4.38 & 0.00 \\
Neighborhood satisfaction & -0.05 & 0.02 & -4.24 & 0.01 \\
Total mediated effect & -0.18 & & & \\
\hline
\end{tabular}

\section{Discussion}

The findings of this study provide evidence of a strong relationship between neighborhood-based experiences and functioning in community settings for person with SMI. Perceptions of neighborhood social climate (i.e., believing that one belongs and is accepted in the neighborhood) were significantly related to self-reported psychiatric distress. These findings support the value of understanding the community-based experience of persons with SMI to promote their adaptive functioning, recovery, and participation in community life (Yanos 2007).

As hypothesized, results indicate that there was a significant positive relationship between each of the neighborhood experience factors and neighborhood social climate. Interestingly, this finding is consistent in the neighborhood literature with those of persons not having SMI (e.g., Unger and Wandersman 1985). Not surprisingly, neighbor relations accounted for more of the variance in neighborhood social climate than satisfaction or safety.

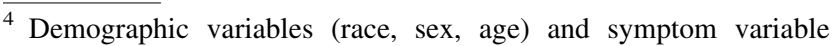
(psychotic and paranoid) have been controlled for in each step but are not reported here in the interest of space and clarity.
}

This finding suggests that individuals' interactions with neighbors had more influence on their perceptions of positive neighborhood social climate than the level of safety or their satisfaction with the neighborhood.

Results also suggest that perceptions of neighborhood social climate are predictive of participants' psychological well-being, as measured by self-reported psychiatric distress. It appears that the more those individuals feel they belong and are accepted in their neighborhoods, the less psychiatric distress they report. This finding is important for individuals with SMI living in community settings because it highlights a potential mechanism to decrease the deleterious effects of psychiatric symptomatology and social rejection to support processes of recovering from mental illness (Carling 1995; Nelson et al. 2001; Kloos 2005). The findings of this study also help to reinforce the assertion of Faris and Dunham (1939) that social connections are vital to the well-being of individuals with serious mental illness.

Finally, the mediation analysis suggests that neighborhood social climate fully mediates (i.e., is responsible for) the relationship between the neighborhood factors and psychological well-being. As hypothesized, it appears that the perceptions of neighborhood social climate, rather than the assessments of neighborhood experience factors, directly accounts for residents' well-being. While individuals may enjoy interacting with neighbors, perceive the neighborhood to be safe, and be satisfied with where they live, it is the accumulated experience of a supportive neighborhood social climate that results from these individual components that actually impacts their well-being. This is consistent with social ecology theory (Moos 1976; Kloos and Shah in press). Thus, it appears to be pragmatic for supported housing or community integration-focused 
programs to bolster neighbor relations, promote experiences of neighborhood safety, and increase neighborhood satisfaction for persons with SMI living in community settings. However, these results strongly suggest that programs evaluate whether persons with SMI actually have enhanced perceptions of positive neighborhood social climate over time.

Although these findings are not entirely novel in the neighborhood literature, they are among the first to document these relationships for a SMI sample. The findings empirically support previous arguments to study neighborhood experiences among members with SMI to promote the functioning of individuals with SMI living in community settings (Carling 1995; Wong and Solomon 2002; Yanos 2007). It is interesting that the findings reported here revealed similar results to those from research with nonmentally ill participants. That is, in this sample, perceptions of neighborhood experiences were related to social climate and well-being in the same direction in which they have been shown to be related in other populations (Aneshensel and Sucoff 1996; Farrell et al. 2004; Prezza et al. 2001; Zeldin and Topitzes 2002). Additionally, because we controlled for both paranoid and psychotic symptoms, individual functioning and symptomatology did not appear to affect substantially the perceptions of neighborhood experiences in such a manner as would confound the results of this study. This does not mean that it is appropriate to generalize all neighborhood research findings from non-mentally ill populations to people with SMI; but it does suggest that processes for belonging and acceptance in community settings may have more similarities across populations than is often assumed.

\section{A Note on Perceptions of Environment}

This study relied on individuals' perceptions of neighborhood phenomena to answer the research questions. There is evidence that perceptions of environments and neighborhood experiences are better predictors of health-related outcomes than objective measures, such as ratings of the physical appearance of the neighborhood, census information on crowding and SES, etc. (e.g., Stiffman et al. 1999; Wright and Kloos 2007). The evaluative process involved in perception may be key to the psychological mechanisms affecting behavior in community settings. However, a potential problem with basing research findings on peoples' perceptions is that they are subjectivepotentially unique to the experiences of the participantand of questionable authority to be generalized to others. Objective measures such as researcher ratings of housing quality, neighborhood crime statistics, and census data can allow for better comparisons between settings. However, such objective ratings have methodological sampling problems when compared to neighborhood experiences. They rely on limited periods of observation, usually using a single rating, and cannot take into account the range of situations and events that can affect neighborhood experience. Thus, they likely do not observe all events that can affect neighborhood experience and likely make observations that are convenient to researchers but not necessarily from time periods when critical incidents are likely to occur. Observer ratings and perceptions by neighborhood residents each have serious limitations.

The methodological dilemma of understanding neighborhood environments is compounded when investigating neighborhood environments and experiences of participants who have SMI because people are often skeptical of the accuracy of their perceptions (Newman 1994). For example, individuals who provided lower reports of the qualities of their neighborhood experiences may have done so because of their potentially distorted attitudes and perceptions of the world. However, the fact that the findings from this study are similar to findings from non-mentally ill populations lends credence to the accuracy of housing and neighborhood perceptions among individuals with SMI living in supported housing. This result is similar to Newman's (1994) finding that individuals with SMI living in community-based apartments were valid reporters of adequate conditions in their homes and neighborhoods. However, Newman also found that persons with SMI tended to minimize the inadequacy of living conditions. The implication of this finding for the current study is that individuals may have underreported deleterious social conditions (e.g., crime in neighborhoods, poor neighbor relations, and lack of acceptance by neighbors). Thus, these findings may paint a positively biased picture of neighbor relations, neighborhood safety, and neighborhood satisfaction as they relate to neighborhood social climate and well-being.

These methodological dilemmas in understanding housing environments and neighborhood experiences of persons with SMI underscore the need for multi-method studies. In the literature we reviewed, research about neighborhood and housing tended to emphasize different disciplinary approaches (e.g., sociology, psychiatry, community psychology) to understanding the potential influence of environmental factors and experiences on functioning. These research approaches were rarely combined, although they can contribute to a complementary investigation. We suggest future research more explicitly compare these different methods to understand experiences in the settings. Future interdisciplinary collaboration could better understand these experiences, disentangle potentially recursive influences, and better establish the relevance of housing environments and experiences in neighborhoods for mental health services and policy. 


\section{Limitations}

Conclusions from the study need to be qualified because of several limitations of method. First, a cross-sectional, nonexperimental design was employed, and individuals were not randomly assigned to a control or treatment group. Thus, causation cannot be inferred from the results. While the theory guiding the research assumed that that the neighborhood experience factors influenced neighborhood social climate, which in turn caused psychological wellbeing, the current study design cannot test this. It could be that the reverse is true: individuals' perceptions of their neighbor relations, neighborhood safety, and neighborhood satisfaction may have resulted from their perceptions of neighborhood social climate, which in turn may have been impacted by their levels of psychiatric distress. Difficulty in assuming causality may also have implications for the mediation analysis in this study. According to the specification error, the conclusions from a mediation analysis are only valid if the causal assumptions are valid. Longitudinal designs and research focused on the experiences of persons with SMI as they move into housing are needed.

A second limitation in the study is the potential for overlapping influence between the Brief Symptom Inventory (BSI) subscales used as covariates in the analyses and the BSI-GSI composite score used as a proxy for psychiatric distress. We used two BSI subscales to control for the influence of psychotic and paranoid symptoms on participants reports of neighborhood experiences. The ten items included in these subscales are also included in the BSIGSI composite score, introducing a threat of inflating relationships between the variables under review and the BSI-GSI composite score. However, we see this limitation as being relatively minor for three reasons. First, we closely compared findings between the models that included the BSI subscales as control variables and those that did not and noted that our primary findings remained the same. This suggests that the possible inflation of relationships with the BSI-GSI outcome variable was not large enough to be problematic. Second, psychotic and paranoid symptoms could be represented twice in analyses (i.e., once as subscales and another time in the BSI-GSI composite score) only if participants endorsed these symptoms as being distressing in the past 30 days. Finally, we were not using the BSI paranoid and psychotic subscales to directly predict BSI-GSI scores, but rather to control for the influence of these symptoms on the neighborhood variables. Thus, we felt that this limitation was less central to the interpretation of our primary findings.

Finally, generalization of conclusions from the study is quite limited. First, the study focused on the housing and neighborhood experiences of persons with SMI living in supported housing. These programs provide a housing subsidy that makes quality housing more attainable. The vast majority of persons with SMI living in community settings but do not have housing subsidies. Second, these data come from a state in the southeastern U.S. Housing and neighborhood experiences in other regions of the U.S. and other countries could have important differences. The sample of this study included persons living in urban, suburban, and rural areas, although urban Charleston and New York City are quite different. The study is best viewed as establishing the relevance of these research questions and provisional findings about housing experiences and psychiatric distress that warrant investigation in other locations.

\section{Future Directions and Conclusions}

An exciting avenue for future research is to link the current findings with research from the community integration field. Community integration is emerging as a priority for efforts to enhance the ability of individuals with SMI to achieve physical, social, and psychological integration in their living environments (e.g., Nelson et al. 2001; Gulcer et al. 2007; Yanos 2007). The current findings are especially relevant to experiences of psychological integration. Psychological integration has been defined as an individual's sense of community or belonging (Aubry and Myner 1996; Wong and Solomon 2002; Prince and Gerber 2005). The neighborhood social climate mediator that we analyzed in the current study measures perceptions of acceptance, belonging, and tolerance in the neighborhood. We found that neighborhood social climate fully mediated the relationship between key neighborhood experiences and psychiatric distress, thus pointing to the important role that psychological integration plays in individuals' adaptation to community life. This speaks to the potential benefit for mental health services to guide interventions aimed at fostering a sense of community among fellow consumers in community-based settings, while also enhancing opportunities for meaningful interactions with non-mentally ill neighbors (Wright and Kloos 2007; Townley et al. 2009).

In conclusion, these findings suggest that experiences of positive neighbor relations, perceptions of neighborhood safety, and satisfaction with the neighborhood are important for individuals to feel that they belong and are accepted by neighbors. These findings can inform the development new approaches for fostering positive neighboring experiences and new models of training staff to facilitate positive interactions (e.g., training staff or peer providers to be community guides and coaches). Such innovative mental health services would help build individual capacities. However, the findings also imply environmental interventions have an important role in creating more opportunities for positive interactions and improved 
personal capacities; neighborhood belonging experiences have an impact on psychological well-being. For example, mental health systems could focus on building relationships with landlords or facilitating participation in a neighborhood-based tenant organization (Kloos et al. 2002). Finally, the findings suggest that neighborhood social climate appears to mediate the relationship between key neighborhood experiences and well-being. These findings suggest that neighborhood social climate could be a vital feature in creating positive community-based experiences and a critical index for maintaining healthy, supportive experiences for individuals with serious mental illness living in community settings.

Acknowledgments Preparation of this manuscript was supported by funding from the National Institute of Mental Health-K23MH65439. Thanks are extended to the staff and consumers of the South Carolina Department of Mental Health, who made this research possible. We would also like to thank M. Lee Van Horn, Psychology Department, University of South Carolina, for his assistance with statistical analyses.

\section{References}

Aneshensel, C. S., \& Sucoff, C. A. (1996). The neighborhood context of adolescent mental health. Journal of Health and Social Behavior, 37(4), 293-310.

Aubry, T., \& Myner, J. (1996). Community integration and quality of life: A comparison of persons with psychiatric disabilities in housing programs and community residents who are neighbors. Candian Journal of Mental Health, 15(1), 5-20.

Barnard, J., \& Rubin, D. B. (1999). Small-sample degrees of freedom with multiple imputation. Biometrika, 86(4), 948-955.

Baron, R. M., \& Kenny, D. A. (1986). The moderator-mediator distinction in social-psychological research: Conceptual, strategic, and statistical considerations. Journal of Personality and Social Psychology, 51(6), 1173-1182.

Baumeister, R. F. (1991). Meanings of life. New York: Guilford Press.

Carling, P. J. (1990). Major mental illness, housing, and supports: The promise of community integration. American Psychologist, 45, 969-975.

Carling, P. J. (1993). Housing and supports for persons with mental illness: Emerging approaches to research and practice. Hospital and Community Psychiatry, 44(5), 439-449.

Carling, P. J. (1995). Return to community: Building support systems for people with psychiatric disabilities. New York: Guilford Press.

Caughy, M. O., O'Campo, P. J., \& Mutaner, C. (2003). When being alone might be better: Neighborhood poverty, social capital, and child mental health. Social Science and Medicine, 57, 227-237.

Chavis, D. M., \& Wandersman, A. (1990). Sense of community in the urban environment: A catalyst for participation and community development. American Journal of Community Psychology, 18, $55-81$.

Cohen, J., Cohen, P., West, S. G., \& Aiken, L. S. (2003). Applied multiple regression/correlation analysis for the behavioral sciences (3rd ed.). New Jersey: Lawrence Erlbaum Associates, Publishers.

Cohen, M., \& Somers, S. (1990). Supported housing: Insights from the Robert Wood Johnson foundation program on chronic mental illness. Journal of Psychosocial Rehabilitation, 13, 43-50.
Corrigan, P. W. (2004). On the stigma of mental illness: Practical strategies for research and social change. Washington: American Psychological Association.

Derogatis, L. R. (1993). Brief symptom inventory (BSI) administration, scoring, and procedures manual (4th ed.). Minneapolis: National Computer Systems.

Dickey, B., Gonzalez, O., Latimer, E., Powers, K., Schutt, R., \& Goldfinger, S. (1996). Use of mental health services by formerly homeless adults residing in group and independent housing. Psychiatric Services, 47, 152-158.

Dixon, L. B., \& Osher, F. (1995). Housing persons with chronic mental illness and substance use disorders. In A. F. Lehman \& L. B. Dixon (Eds.), Double Jeopardy: Chronic mental illness and substance use disorders (Vol. 3, pp. 211-228). Philadelphia: Harwood Academic Publishers/Gordon and Breach Science Publishers.

Drake, R. E., Wallach, M. A., Teague, G. B., Freeman, D. H., et al. (1991). Housing instability and homelessness among rural schizophrenic patients. American Journal of Psychiatry, 148, 330-336.

Evans, G. W., Wells, N. M., \& Moch, A. (2003). Housing and mental health: A review of the evidence and a methodological and conceptual critique. Journal of Social Issues, 59(3), 475-500.

Faris, R. E., \& Dunham, H. W. (1939). Mental disorders in urban areas: An ecological study of schizophrenia and other psychoses. Chicago: The University of Chicago Press.

Farrell, S., Aubry, T., \& Coulombe, D. (2004). Neighborhoods and neighbors: Do they contribute to personal well-being? Journal of Community Psychology, 32(1), 9-25.

Fiske, S. T. (2004). Social beings: Core motives in social psychology. Hoboken: John Wiley Sons, Inc.

Goldman, H., \& Morrisey, J. (1985). The alchemy of mental health policy: Homelessness and the fourth cycle of reform. American Journal of Public Health, 75, 727-731.

Grillo, M. C., Teixeira, M. A., \& Wilson, D. C. (2010). Residential satisfaction and civic engagement: Understanding the causes of community participation. Social Indicators Research, 97(3), 451-466.

Gulcer, L., Tsemberis, S., Stefancic, A., \& Greenwood, R. M. (2007) Community integration of adults with psychiatric disabilities and histories of homelessness. Community Mental Health Journal, $43(3), 211-228$.

Kawachi, I. (2002). Social epidemiology. Social Science and Medicine, 54, 1739-1741.

Kloos, B. (2005). Creating new possibilities for promoting liberation, well-being, and recovery: Learning from experiences of psychiatric consumers/survivors. In G. Nelson \& I. Prillitensky (Eds.), Community psychology: In pursuit of well-being and liberation (pp. 426-447). London: MacMillan.

Kloos, B., \& Shah, S. (2009). A social ecological approach to investigating relationships between housing and adaptive functioning for persons with serious mental illness. American Journal of Community Psychology, 44(3-4), 316-326.

Kloos, B., Zimmerman, S. O., Scrimenti, K., \& Crusto, C. (2002). Landlords as partners for promoting success in supported housing: "It takes more than a lease and a key". Psychiatric Rehabilitation Journal, 25, 235-244.

Lam, J. A., \& Rosenheck, R. (1999). Social support and service use among homeless persons with serious mental illness. International Journal of Social Psychiatry, 45, 13-28.

Markowitz, F. E. (1998). The effects of stigma on the psychological well-being and life satisfaction of persons with mental illness. Journal of Health and Social Behavior, 39(4), 335-347.

Martinez, M. L., Black, M., \& Starr, R. H. (2001). Factorial structure of the Perceived Neighborhood Scale (PNS): A test of longitudinal invariance. Journal of Community Psychology, 30(1), $23-43$. 
McMillan, D. W., \& Chavis, D. M. (1986). Sense of community: A definition and theory. Journal of Community Psychology, 14, 6-23.

Moos, R. (1974). Evaluating treatment environments: A social ecological approach. New York: Wiley.

Moos, R. H. (1976). The human context: Environmental determinants of behavior. New York: Wiley.

Nelson, G., Lord, J., \& Ochocka, J. (2001). Shifting the paradigm of community mental health: Towards empowerment and community. Toronto: University of Toronto Press.

Newman, S. J. (1994). The housing and neighborhood conditions of persons with severe mental illness. Hospital and Community Psychiatry, 45(4), 338-343.

Newman, S. (2001). Housing attributes and serious mental illness: Implications for research and practice. Psychiatric Services, 52, 1309-1317.

Newman, S. J., Rechovsky, J. D., Kaneda, K., \& Hendrick, A. M. (1994). The effects of independent living on persons with chronic mental illness: An assessment of the section 8 certificate program. The Milbank Quarterly, 72(1), 171-198.

Pretty, G. H. (1990). Relating psychology sense of community to social climate characteristics. Journal of Community Psychology, $18,60-65$.

Pretty, G. H., Conroy, C., Dugay, J., Fowler, K., \& Williams, D. (1996). Sense of community and its relevance to adolescents of all ages. Journal of Community Psychology, 24(4), 365-379.

Prezza, M., Arrici, M., Roberti, T., \& Tedeschi, G. (2001). Sense of community referred to the whole town: its relations with neighboring, loneliness, life satisfaction, and area of residence. Journal of Community Psychology, 29(1), 29-52.

Prince, P. N., \& Gerber, G. J. (2005). Subjective well-being and community integration among clients of assertive community treatment. Quality of Life Research, 14, 161-169.

Prince, P. N., \& Prince, C. R. (2002). Perceived stigma and community integration among clients of assertive community treatment. Psychiatric Rehabilitation Journal, 25(4), 323-331.

Rog, D. (2004). The evidence on supported housing. Psychiatric Rehabilitation Journal, 27, 324-344.

Ross, C. E., Reynolds, J. R., \& Geis, K. J. (2000). The contingent meaning of neighborhood stability for residents' psychological well-being. American Sociological Review, 65(4), 581-597.

Sampson, R. J. (2003). The neighborhood context of well-being. Perspective in Biology and Medicine, 46(3), S53-S64.

Schafer, J. L. (1999). Multiple imputation: A primer. Statistical Methods in Medical Research, 8, 3-15.

Segal, S. P., Baumohl, J., \& Moyles, E. W. (1980). Neighborhood types and community reaction to the mentally ill: A paradox of intensity. Journal of Health and Social Behavior, 21(4), $345-359$.

Silver, E. (2002). Mental disorder and violent victimization: The mediating effect of involvement in conflicted social relationships. Criminology, 40(1), 191-212.
Silver, E., Mulvey, E. P., \& Swanson, J. W. (2002). Neighborhood structural characteristics and mental disorder: Faris and Dunham revisited. Social Science and Medicine, 55, 1457-1470.

Stiffman, A. R., Hadley-Ives, E., Elze, D., Johnson, S., \& Dore, P. (1999). Impact of environment on adolescent mental health and behavior: Structural equation modeling. American Journal of Orthopsychiatry, 69(1), 73-86.

Townley, G., \& Kloos, B. (2009). Development of a measure of sense of community for individuals with serious mental illness residing in community settings. Journal of Community Psychology, 37(3), 362-380.

Townley, G., Kloos, B., \& Wright, P. A. (2009). Understanding the experience of place: Expanding methods to conceptualize and measure community integration of persons with serious mental illness. Journal of Health and Place, 15(2), 520-531.

Unger, D. G., \& Wandersman, A. (1985). The importance of neighbors: The social, cognitive, and affective component of neighboring. American Journal of Community Psychology, 13, 139-169.

Ware, N. C., Hopper, K., Tugenberg, T., Dickey, B., \& Fisher, D. (2007). Connectedness and citizenship: Redefining social integration. Psychiatric Services, 58(4), 469-474.

Ware, N. C., Hopper, K., Tugenberg, T., Dickey, B., \& Fisher, D. (2008). A theory of social integration as quality of life. Psychiatric Services, 59, 27-33.

Wong, Y.-L. I., \& Solomon, P. (2002). Community integration of persons with psychiatric disabilities in supportive independent housing: Conceptual model and methodological issues. Mental Health Services Research, 4(1), 13-28.

Wright, P. A., \& Kloos, B. (2007). Housing environment and mental health outcomes: A levels of analysis perspective. Journal of Environmental Psychology, 27(1), 79-89.

Yanos, P. T. (2007). Beyond "landscapes of despair": The need for new research on the urban environment, sprawl, and the community integration of persons with serious mental illness. Health and Place, 13, 672-676.

Young, A. F., Russell, A., \& Powers, J. R. (2004). The sense of belonging to a neighborhood: Can it be measured and is it related to health and well-being in older women? Social Science and Medicine, 59, 2627-2637.

Zeldin, S., \& Topitzes, D. (2002). Neighborhood experiences, community connection, and positive beliefs about adolescents among urban adults and youth. Journal of Community Psychology, 30(6), 647-669.

Ziersch, A., Baum, F. E., MacDougall, C., \& Putland, C. (2005). Neighborhood life and social capital: The implications for health. Social Science and Medicine, 60, 71-86. 